Témoigner Témoigner. Entre histoire et mémoire

Getuigen Revue pluridisciplinaire de la Fondation Auschwitz

Violences radicales en scène

\title{
Dantsig Baldaev, Élisabeth Anstett et Luba Jurgenson (dir.), Gardien de camp. Tatouages et dessins du Goulag
}

Genève, Syrtes, 2013

\section{Yoann Sarrat}

\section{(2) OpenEdition \\ Journals}

\section{Édition électronique}

URL : https://journals.openedition.org/temoigner/3711

DOI : 10.4000/temoigner.3711

ISSN : 2506-6390

\section{Éditeur :}

Éditions du Centre d'études et de documentation Mémoire d'Auschwitz, Éditions Kimé

\section{Édition imprimée}

Date de publication : 1 octobre 2015

Pagination : 210-212

ISSN : 2031-4183

Référence électronique

Yoann Sarrat, « Dantsig Baldaev, Élisabeth Anstett et Luba Jurgenson (dir.), Gardien de camp. Tatouages et dessins du Goulag », Témoigner. Entre histoire et mémoire [En ligne], 121 | 2015, mis en ligne le 01 octobre 2016, consulté le 04 février 2022. URL : http://journals.openedition.org/temoigner/3711 ; DOI : https://doi.org/10.4000/temoigner.3711

Ce document a été généré automatiquement le 4 février 2022.

Tous droits réservés 


\title{
Dantsig Baldaev, Élisabeth Anstett et Luba Jurgenson (dir.), Gardien de camp. Tatouages et dessins du Goulag
}

Genève, Syrtes, 2013

\author{
Yoann Sarrat
}

\section{RÉFÉRENCE}

Dantsig Baldaev, Élisabeth Anstett et Luba Jurgenson (dir.), Gardien de camp. Tatouages et dessins du Goulag. traduit du russe par Luba Jurgenson, Genève, Syrtes, 2013, 208 p.

1 Dans la boutique du musée du Quai Branly, lors de l'exposition «Tatoueurs, Tatoués » (présentée de mai 2014 à octobre 2015), nous trouvons cet impressionnant ouvrage, paru en 2013, dirigé par Élisabeth Anstett et Luba Jurgenson : Gardien de camp. Tatouages et dessins du Goulag, comportant un Album de Dantsig Baldaev ainsi que sa traduction française (réalisée par Luba Jurgenson).

2 Cet Album, aux valeurs ethnographique et sociologique incontestables, est celui d'une "mémoire du Goulag en dessins» (p. 7) - réalisés de 1949 à 1989 -, qui adopte un langage graphique polytechnique pour décrire, présenter, systématiser et donner à voir un univers de violences extrêmes diverses, y compris sexuelles. «La présence d'un point de vue omniscient permet à cet égard de souligner que les dessins de Baldaev prennent appui sur une double expérience intime des espaces carcéraux.» (p. 28) L'Album de Dantsig Baldaev "fournit une source documentaire irremplaçable sur l'organisation spatiale de l'institution concentrationnaire soviétique, une source d'autant plus précieuse qu'il n'existe que très peu de photographies ou de films permettant de visualiser l'intérieur du Goulag"(p.27) où se côtoient plusieurs catégories de zeks (détenus) dont les « ennemis du peuple » et des criminels de la pègre. Dantsig Baldaev était un «officier du Ministère de l'Intérieur soviétique, longtemps membre de l'institution pénitentiaire» (p.7) et fils d'un ethnographe. Il est aussi 
l'auteur d'autres ouvrages du même type, dont Russiana criminal Tattoo, Encyclopedia, en trois volumes, réalisés avec Sergueï Vasiliev.

4 Cet ouvrage, très riche dans sa forme comme dans son contenu, contient d'abord plusieurs articles introductifs et présentatifs écrits par les deux directrices de l'édition. Ils s'intéressent à l'Album en tant qu'objet polymorphique et dont Luba Jurgenson commente la valeur polyphonique : «Album du gardien » (p. 7), « répertoire graphique de l'horreur » (p. 8), « album photographique » (p. 13), «bande dessinée » (bâtie sur le modèle des komiksy, comme sont nommées les bandes dessinées en Russie et du loubok, désignant des dessins populaires avec de courtes légendes) (p. 15), «scrapbook» (p. 17), etc. Autant de formes au service d'un "produit de son temps " en même temps qu'un "geste transgressif» (p.16). Se pose aussi la question, dans les textes d'Anstett et Jurgenson, de savoir si cet Album est une œuvre d'Art brut.

5 Il est très intéressant que cet ouvrage s'inscrive dans le corpus d'œuvres présentées dans la boutique de l'exposition du musée du Quai Branly, tout d'abord parce qu'il répond efficacement aux œuvres exposées qui insistent sur la valeur des tatouages comme porteurs de toute une mémoire, à la fois biographique et historique, mais aussi parce qu'il permet de nouvelles approches et problématiques. L'exposition rend très bien compte de l'évolution de cette pratique artistique qu'est le tatouage, mais il est nécessaire, semble-t-il, d'insister sur la mémoire des camps et des prisons par le tatouage et de rappeler sans cesse qu'il a et a eu une place sociale très forte, faisant entièrement partie de l'univers, du langage, et de la «culture » - et Luba Jurgenson revient d'ailleurs sur les problématiques et enjeux de cette notion quand elle est mise en lien avec le Goulag - des camps divers des violences extrêmes et des exterminations de masses du XXe siècle, mais aussi, et le travail de Dantsig Baldaev est également remarquable sur ce point, de l'univers, langage et culture des criminels de tous genres. «On voit bien [...] comment l'expérience du territoire-Goulag vient instaurer un monde. » (p. 29)

6 La peau est continuellement attaquée dans l'univers concentrationnaire, que ce soit au Goulag ou dans le camp de concentration et d'extermination : par les lavements divers, les douches, les tatouages-marquages à l'entrée, les tortures, les affaiblissements corporels liés à la faim, à la maladie; le tatouage "artistique ", volontaire, s'inscrit dans un processus de défense personnel, de révolte corporelle qui refuse ces attaques multiples et qui cherche à ré-individualiser la peau, à se la réapproprier malgré la condition concentrationnaire et les enfermements. Il est alors comme un message adressé à soi-même et aux autres (message qui peut être préjudiciable, le travail de Baldaev le présente bien à plusieurs reprises). Le geste de la peau trouée puis tatouée répond et fait écho aux gestes d'attaques répétées de la corporéité de l'être enfermé. On suppose également que le tatouage est une prise de risque volontaire dans le camp ou la prison où les conditions d'hygiène sont déplorables, prise de risque qui fait aussi partie de ce processus d'individualisation et de réinvestissement identitaire. En somme, nous le savons, et l'exposition tout comme l'ouvrage le rappellent bien, la valeur d'un tatouage réalisé dans certaines conditions, de vie, d'hygiène, d'enfermement, est immense et quasi métaphorique: elle est une adresse au monde, intérieur comme extérieur.

7 Le tatouage est biographique, il est une "archive de soi » (p. 34) mais il est aussi symbolique, métonymique et allégorique : il constitue une image forte qui exprime une idée plus vaste et importante. Il a aussi souvent pour « vocation de figurer des états 
d'âme, des dispositions intérieures " (p. 44). Ainsi nous lisons à travers le tatouage, qui est une «carte de visite» (p. 53), ces conditions, les modalités de ce monde, etc. Il est par ailleurs porteur d'un passé ineffaçable lorsqu'il est réalisé à l'extérieur et avant l'expérience concentrationnaire. Le travail de Baldaev implique très nettement ce rapport, « corps et biographie ont intimement partie liée. La surface du corps devient le support sur lequel s'écrivent à la fois la vie et l'identité du détenu» (p. 34). Élisabeth Anstett rappelle également, évoquant la philosophie de Merleau-Ponty, que «le corps est instrument général de la compréhension du monde. » (p. 34)

Ce travail de mémoire par les dessins et les tatouages constitue un objet rare de témoignage renforcé par la position particulière de ce témoin - artiste, en l'occurrence, dont les dessins révèlent des partis pris esthétiques très intéressants - qu'est Dantsig Baldaev. Les dessins sont très puissants, intenses et explicites, pouvant évoquer ou rappeler les univers graphiques d'Otto Dix, de David olère ou encore de Walter Spitzer, voire même d'Art Spiegelman sans l'anthropomorphisme, donc par exemple dans des œuvres comme Prisonnier sur la planète enfer. Dans les dessins de la " chaîne de la mort " (p. 85), d'une très grande violence, on remarquera le travail sur les visages et les mains, lieux significatifs et représentatifs des affres et tortures diverses, ceux des gardes et autres officiers oscillent entre sadisme, moquerie et sévérité extrêmes. Ceux des « ennemis du peuple» arrêtés par le NKVD, des torturés, détenus, déportés, des zeks, révèlent toute l'horreur subie, les mains apparaissant souvent en signe de protection maladroite de ce qu'il reste d'intimité et de dignité (voir par exemple la reproduction très dérangeante $\mathrm{p}$. 11). Le travail de Baldaev constitue, selon ses dires, « un bref rappel des terribles décennies au cours desquelles le déchainement de la bêtise, de la cruauté et de la médiocrité emporta trente-six millions de vies, la fine fleur des peuples de l'URSS. » (p. 97)

9 L'œuvre montre à nouveau que la bande dessinée, dans sa conjugaison parfaite du texte et de l'image, possède une valeur esthétique forte, elle est sensationnelle, elle a un impact direct sur le lectorat. Celles de Baldaev sont visuellement impressionnantes, parfois choquantes, mais toujours animées d'une efficience renforcée par les commentaires accompagnateurs.

L'ouvrage permet donc de découvrir le travail de Dantsig Baldaev sans en faire l'apologie, mais en posant des questions légitimes (par exemple sur la position voyeuriste de l'artiste), il permet aussi d'ouvrir diverses perspectives grâce aux très bons textes d'Anstett et Jurgenson, de proposer différentes approches et d'appeler à poursuivre les recherches (principalement en histoire de l'art). 